\title{
One-Pot Synthesis of 5-Arylpent-4-enoate Derivatives from Baylis-Hillman Acetates: Use of Phosphorous Ylide
}

\author{
Yang Jin Im, Jeong Eun Na, and Jae Nyoung Kim*

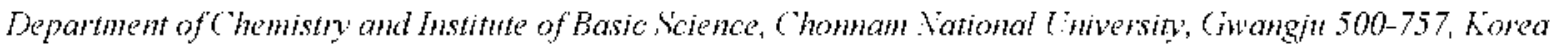 \\ Received December 5,2002
}

Key Words : 5-Any lpent-4-enoalıs, Baylis-Hillman acelatex, Phosphonium salts, Hỵdrolỵsis

Basavaiah et al. have published some papers dealing with the Jolunson-Claisen rearrangement of the Baylis-Hillman adducts in order to prepare 5-arylpent-t-enoates or 4cyanoalk-t-enoates.' Shen ef al. have also reported the synthesis of the latter compounds by using the sequential Michael reaction and Homer-Wadworth-Emmons (HWE) reaction of phosphonates.' Recently: we have also reported the synthesis of 5-arylpent-t-enoates from the BaylisHillman acetates. ${ }^{3}$ The reaction was carried out wa the tandem $\mathrm{S}_{\aleph} 2^{\prime}$ reaction of diethyl malonate and subsequent decarbethoxylation process. However, the decarbethoxylation step required long reaction time (2-6 days) and high temperature (xylene. reflux) ${ }^{34}$ Thus. mild reaction conditions were needed.

Recently Zaragoza reported one-step conversion of alcohols into nitriles with simultaneous two-carbon chain elongation by using (cyanomethyl)trimethylphosphonium iodide. ${ }^{5}$ In the reaction. alcohols were converted into the corresponding iodides and react with the ylide to generate the corresponding alkylated phosphonium salts. Final hydrolysis with aqueous base furnished the desired products. ${ }^{.}$it is well known that phosphonium salt can be hydrolysed to the lyy drocarbon analog. ${ }^{6}$

ln this respect. we emsisioned that if the reaction of the Baylis-Hillman acetate and appropriate phosphorous ylide would produce the corresponding phosphonium salt wa the $\mathrm{S}_{*} 2^{\prime}$ type mechanism. we could prepare desired 5-arylpent4-enoates. The reaction of the Baylis-Hillman acetate and phosphorous ylide has not been reported to the best of our knowledge. Thus. we examined the possibility and report herein an efficient synthetic method for the synthesis of 5arylpent-t-enoate derivatives.

As shown in Scheme 1, the reaction of the Baylis-Hillman acetate 1a and (carbethoxy methylene)tripheny lphosphorane (2a) in THF gave the phosphonium salt 3a. We used the reaction mixture directly in the next hydrolysis step without further purification. Following hydrolysis of phosphonium salt 3 a was examined by using various conditions. ${ }^{5 \%}$ The use of aqueous $\mathrm{KCN}$ gave the best results $(90 \%$ for $4 a$ ). Instead. the use of aqueous $\mathrm{NaHCO}_{3}(84 \%)$ or aqueous KI solution $(64 \%)$ afforded $4 a$ in lower yields (Table 1$)$. The structure of ta was exclusively $E$-form as in our previously paper.

The representative results for the synthesis of 5 -arylpent+-enoates. 4a-y. are summarized in Table 1. Baylis-Hillman acetates 1a-d (derived from ethyl acrylate) and 1e-f (derived from acrylonitrile) were used as substrates. In all cases we could obtain the desired products $4 \mathbf{a}-\mathrm{g}$ in $25-90 \%$ isolated yields. For the nitrile-substituted Bay lis-Hillman acetates 1e and $1 \mathrm{f}$. the obtained products $\mathbf{4 f}$ and $\mathbf{4 g}$ were the mixtures of $E$ and $Z$ isomers. Another ylide. 1-triphenylphosphoranylidene-2-propanone (2b), gave the corresponding product $4 \mathbf{c}$. albeite. in low yield. In this case we could not obtain the desired product by following the usual reaction sequence. The best result $(25 \%)$ was obtained by simply mixing 1 a and 2b in DMF and heating the reaction mixture for $25 \mathrm{~h}$.

The reaction mechamism could be proposed as shown in Scheme 2. The reaction of 1 a and $2 \mathbf{a}$ in THF gave the corresponding phospholuium salt 3a (1ikle supro) wia the addition-elimination process. Attack of cranide ion to the phosphorous atom leaved ester enolate. which was protonated to give the product $4 \mathbf{a}$.

ln conclusion we disclosed a facile synthetic method for the preparation of synthetically useful 5-arylpent-t-enoate derivatives. This procedure has some merits over the previous method ${ }^{3}$ in the respects of (i) mild reaction conditions and (ii) one-pot reaction.

\section{Experimental Section}

All materials and solvents were of reagent grade as received from commercial sources. Baylis-Hillman adducts and their acetates were prepared as reported?<smiles>C=C(OCC)C(OC(C)=O)c1ccccc1</smiles>

1a<smiles>CCOC(=O)C=Pc1ccccc1</smiles>

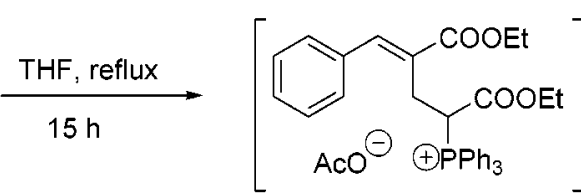

3a

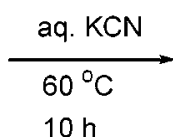

$10 \mathrm{~h}$

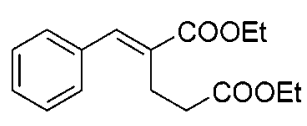

$4 a$

Scheme 1 
Table 1. Sỵthesis of 5-arylpent-4-enoate derivatives ta-g

\begin{tabular}{|c|c|c|c|c|}
\hline$\overline{\text { Entry }}$ & Sulbstrate 1 & Conditions & Product 2 & Yicld $\left({ }^{\circ} 0\right)$ \\
\hline 1 & $1 a$ & $\begin{array}{l}\text { 1. } \mathrm{Ph}_{3} \mathrm{P}=\mathrm{CHCOOEt}(2 \mathrm{a}, 1.0 \text { equiv) } \\
\text { THF, reflux, } 15 \mathrm{~h} \\
\text { 2. aq. } \mathrm{KCN}(1.0 \text { equiv) } \\
60^{\circ} \mathrm{C}, 10 \mathrm{~h}\end{array}$ & 4 & $90^{\mathrm{a}}$ \\
\hline 2 & $1 a$ & $\begin{array}{l}\text { 1. same as in entry } 1 \\
\text { 2. aq. } \mathrm{NaHCO}_{3}(2.0 \text { equiv) } \\
60^{\circ} \mathrm{C}, 13 \mathrm{~h}\end{array}$ & $4 a$ & $84^{\mathrm{a}}$ \\
\hline 3 & $1 \mathrm{a}$ & $\begin{array}{l}\text { 1. same as in entry } 1 \\
\text { 2. aq. } \mathrm{KI}(3.0 \text { equiv) } \\
60^{\circ} \mathrm{C}, 10 \mathrm{~h}\end{array}$ & $4 a$ & $64^{2}$ \\
\hline 4 & $1 b$ & $\begin{array}{l}\text { 1. 2a (1.0 equiv) } \\
\text { THF, reflux, } 24 \mathrm{~h} \\
\text { 2. aq. } \mathrm{KCN} \text { (1.0 equiv) } \\
60^{\circ} \mathrm{C}, 18 \mathrm{~h}\end{array}$ & & $60^{a}$ \\
\hline 5 & $1 c$ & $\begin{array}{l}\text { 1. } 2 \mathrm{a} \text { (1.0 equiv) } \\
\text { THF, reflux, } 24 \mathrm{~h} \\
\text { 2. aq. KCN ( } 1.0 \text { equiv) } \\
60^{\circ} \mathrm{C}, 18 \mathrm{~h}\end{array}$ & & $64^{a}$ \\
\hline 6 & OAC & $\begin{array}{l}\text { 1. } 2 \mathrm{a} \text { ( } 1.0 \text { equiv) } \\
\mathrm{THF}, \text { reflux }, 24 \mathrm{~h} \\
\text { 2. aq. } \mathrm{KCN}(1.0 \text { equiv) } \\
60^{\circ} \mathrm{C}, 18 \mathrm{~h}\end{array}$ & SMe & $74^{a}$ \\
\hline 7 & $1 a$ & $\begin{array}{l}\mathrm{Ph}_{3} \mathrm{P}=\mathrm{CHCOCH}_{3}(2 \mathrm{~b}, 1.0 \text { equiv }) \\
\text { DMF, } 110^{\circ} \mathrm{C}, 25 \mathrm{~h}\end{array}$ & $4 e$ & $25^{\mathrm{a}}$ \\
\hline 8 & $1 \mathrm{e}$ & $\begin{array}{l}\text { 1. } 2 \mathrm{a}(1.0 \text { equiv) } \\
\text { THF, reflux }, 24 \mathrm{~h} \\
\text { 2. aq. } \mathrm{KCN}(1.0 \text { equiv) } \\
60^{\circ} \mathrm{C}, 24 \mathrm{~h}\end{array}$ & $\mathbf{4 f}$ & $84^{b}$ \\
\hline 9 & OAC & $\begin{array}{l}\text { 1. 2a (1.0 equiv) } \\
\text { THF, reflux, } 24 \mathrm{~h} \\
\text { 2. aq. } \mathrm{KCN}(1.0 \text { equiv) } \\
60^{\circ} \mathrm{C}, 22 \mathrm{~h}\end{array}$ & & $76^{\mathrm{b}}$ \\
\hline
\end{tabular}

"Pure $E$-isomer. Traec amounts of $Z$-isomer was el iminated duting column purification process. " $E Z-1: 3$ mixtures.

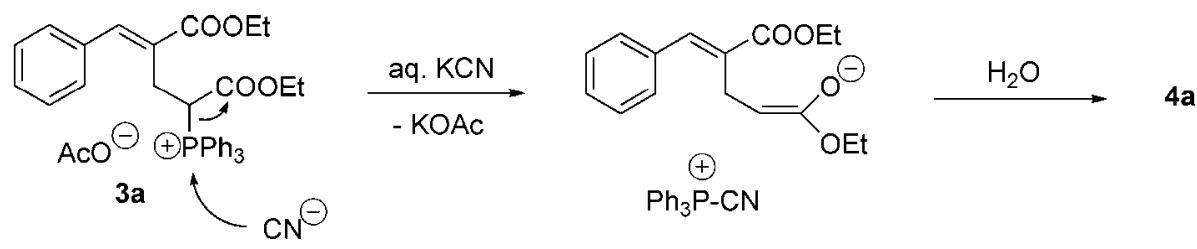

Scheme 2

A typical procedure for the synthesis of $4 \mathrm{a}$ is as follows: To a stirred solution of 1a $(496 \mathrm{mg} .2 .0 \mathrm{mmol})$ and (carbethoxymethylene)triphenylphosphorane (2a. $696 \mathrm{mg}$. $2.0 \mathrm{mmol}$ ) in THF (10 $\mathrm{mL}$ ) was heated to reflux for $15 \mathrm{~h}$.
Aqueous solution of $\mathrm{KCN}(130 \mathrm{mg} .2 .0 \mathrm{mmol}$ in $5 \mathrm{~mL}$ of $\mathrm{H}_{3} \mathrm{O}$ ) was added and stirred at $60{ }^{\circ} \mathrm{C}$ for $10 \mathrm{~h}$. After usual workup and column chromatographic purification (hexane/ ether. 8: 1) 4a was obtained as clear oil. $498 \mathrm{mg}(90 \%)$. 
Selected data for ta: ${ }^{3}$ oil: IR $(\mathrm{KBr}) 173+.1707 \mathrm{~cm}^{-1}:{ }^{1} \mathrm{H}$ NMR $\left(\mathrm{CDCl}_{3}\right) \delta 1.15(\mathrm{t} . J=7.2 \mathrm{~Hz} .3 \mathrm{H}), 1.27(\mathrm{t} . J=7.2 \mathrm{~Hz}$. $3 \mathrm{H}) .2 .45-2.5 \mathrm{l}(\mathrm{m}, 2 \mathrm{H}) .2 .77-2.83(\mathrm{~m}, 2 \mathrm{H}) .+.03(\mathrm{q} . J=7.2$ Hz. $2 \mathrm{H}), 4.20(\mathrm{q} . J=7.2 \mathrm{~Hz}, 2 \mathrm{H}), 7.27-7.32(\mathrm{~m} .5 \mathrm{H}) .7 .65(\mathrm{~s}$. $1 \mathrm{H}):{ }^{1.3} \mathrm{C}$ NMR $\left(\mathrm{CDCl}_{3}\right) \delta 14.07 .14 .19 .22 .99 .33 .44 .60 .32$. $60.81,128.47,128.49,129.05,131.38,135.21,140.01$. 167.69. 172.61.

Spectroscopic data of other compounds are as follows. 4b (E): oil: IR (KBr) 2981. 1732. 1709, 1250. $1092 \mathrm{~cm}^{-1}:{ }^{1} \mathrm{H}$ NMR $\left(\mathrm{CDCl}_{3}\right) \delta 1.2+(\mathrm{t} . J=7.2 \mathrm{~Hz} .3 \mathrm{H}), 1.35(\mathrm{t} . J=7.2 \mathrm{~Hz}$, $3 \mathrm{H}) .2 .5+(\mathrm{t} . J=8.0 \mathrm{~Hz} .2 \mathrm{H}) .2 .8+$ (t. $J=8.0 \mathrm{~Hz} .2 \mathrm{H}) .4 .1 \mathrm{l}$ (q. $J=7.2 \mathrm{~Hz} .2 \mathrm{H}) .4 .28(\mathrm{q} . J=7.2 \mathrm{~Hz} .2 \mathrm{H}) .7 .31$ (d. $J=8.5$ Hz. $2 \mathrm{H}$ ). 7.38 (d. $J=8.5 \mathrm{~Hz} .2 \mathrm{H}) .7 .66(\mathrm{~s}, 1 \mathrm{H}) ;{ }^{1.3} \mathrm{C}$ NMR ( $\left.\mathrm{CDCl}_{3}\right) \delta 14.15,14.24,23.00,33.35,60.48,61.01,128.82$. 130.41. 132.05. 133.68, 134.49, 138.69, 167.51, 172.56; Mass (70 eV) $m z$ (rel. intensity) 129 (61). 163 (100), 236 (99). $26+(69) .310(\mathrm{M}, 21), 312\left(\mathrm{M}^{\prime}+2.70\right) .+\mathrm{c}(E)$ : oil: IR (KBr) $1739,1705,1254 \mathrm{~cm}^{-1}:{ }^{1} \mathrm{H}$ NMR $\left(\mathrm{CDCl}_{3}\right) \delta 1.2+$ (t. $J$ $=7.2 \mathrm{~Hz} .3 \mathrm{H}) .1 .35(\mathrm{t} . J=7.2 \mathrm{~Hz}, 3 \mathrm{H}), 2.53-2.58(\mathrm{~m} .2 \mathrm{H})$. $2.86-2.92$ (m. $2 \mathrm{H}) .4 .1+(\mathrm{q} . J=7.2 \mathrm{~Hz}, 2 \mathrm{H}), 4.27$ (q. $J=7.2$ Hz. $2 \mathrm{H}), 7.20(\mathrm{~d} . J=8.1 \mathrm{~Hz} .2 \mathrm{H}) .7 .29(\mathrm{~d} . J=8.1 \mathrm{~Hz}, 2 \mathrm{H})$. 7.70 (s. $1 \mathrm{H}),{ }^{1.3} \mathrm{C}$ NMR $\left(\mathrm{CDCl}_{3}\right) \delta 14.15,14.28 .21 .28$, $23.08,33.49 .60 .39,60.83,129.24,129.30 .130 .52 .132 .37$. 138.74. 140.09. 167.94. 172.79: Mass (70 eV) $m z$ (rel. intensity) $115(25), 129(43) .143(100), 188(+7) .216(46)$. $24+(40) .290\left(\mathrm{M}^{\prime} .16\right)$. td (E): oil: IR (KBr) 2981. 1732. $1709.1238 \mathrm{~cm}^{-1}:{ }^{1} \mathrm{H}$ NMR $\left(\mathrm{CDCl}_{5}\right) \delta 1.14(\mathrm{t}, J=7.2 \mathrm{~Hz}$. $3 \mathrm{H}$ ). 1.26 (t. $J=7.2 \mathrm{~Hz}, 3 \mathrm{H}$ ), $2.45-2.50$ (m. $2 \mathrm{H}$ ). $2.77-2.82$ (m. $2 \mathrm{H}) .3 .73$ (s. $3 \mathrm{H}), 4.02$ (q. $J=7.2 \mathrm{~Hz} .2 \mathrm{H}$ ). 4.19 (q. $J=$ $7.2 \mathrm{~Hz}, 2 \mathrm{H}) .6 .78-6.88(\mathrm{~m}, 3 \mathrm{H}) .7 .22$ (t. $J=7.8 \mathrm{~Hz}, \mathrm{lH}) .7 .6 \mathrm{l}$ (s. 1H): ${ }^{2} \mathrm{C}$ NMR $\left(\mathrm{CDCl}_{3}\right) \delta 14.13,14.26,23.16,33.54$. $55.21,60 .+1,60.91,11+.29,11+.36 .121 .52 .129 .57,131.66$, 136.58. 139.99. 159.58. 167.74. 172.69: Mass $(70 \mathrm{eV}) \mathrm{mz}$ (rel. intensity) 115 (20). 159 (100). 215 (27). 260 (25). 306 $\left(\mathrm{M}^{\prime}, 16\right)$. 4c $(E)$ : oil: $\mathrm{IR}(\mathrm{KBr}) 2970,1701,1250 \mathrm{~cm}^{-1}$ : ${ }^{1} \mathrm{H}$ NMR $\left(\mathrm{CDCl}_{3}\right) \delta 1.35$ (t. $\left.J=7.2 \mathrm{~Hz} .3 \mathrm{H}\right), 2.15$ (s. $\left.3 \mathrm{H}\right)$. $2.65-2.72(\mathrm{~m}, 2 \mathrm{H}) .2 .78-2.84(\mathrm{~m}, 2 \mathrm{H}) .4 .28$ (q. $J=7.2 \mathrm{~Hz}$. 2H). 7.32-7.4l (m. 4H), 7.72 (s. 1H); Mass $(70 \mathrm{eV}) \mathrm{mz}$ (rel. intensity) $43(6+), 115(50), 129(100) .157(90) .200$ (75). $246\left(\mathrm{M}^{\prime} .10\right)$. \&f ( $\left.E \mid Z\right)$ : oil: IR (KBr) 2981. 2210 . 1736. $1227 \mathrm{~cm}^{-1}: E$-form: 'H NMR $\left(\mathrm{CDCl}_{3}\right) \delta 1.25$ (t. $J=7.2 \mathrm{~Hz} .3 \mathrm{H}) .2 .64-2.85(\mathrm{~m} .4 \mathrm{H}) .+1+(\mathrm{q} . J=7.2 \mathrm{~Hz}, 2 \mathrm{H})$. $7.27-7.73(\mathrm{~m}, 6 \mathrm{H}) . \angle$-form: ' $\mathrm{H}$ NMR $\left(\mathrm{CDCl}_{3}\right) \delta 1.26(\mathrm{t}$. $J=7.2 \mathrm{~Hz}, 3 \mathrm{H}) .2 .64-2.85(\mathrm{~m} .4 \mathrm{H}),+.15(\mathrm{q} . J=7.2 \mathrm{~Hz}, 2 \mathrm{H})$. 7.03 (s. $1 \mathrm{H}) .7 .27-7.73$ (m. $5 \mathrm{H}), 7.70-7.73$ (m. 2H): Mass (70 eV) $m z$ (rel. intensity) 115 (37). 155 (100). 184 (16). 229 (M . 30). 4g ( $E \cdot Z)$ : oil: IR (KBr) 2981, $2210,1732$.
$1184 \mathrm{~cm}^{-1}: E$-form: ${ }^{1} \mathrm{H} \mathrm{NMR}\left(\mathrm{CDCl}_{3}\right) \delta 1.18(\mathrm{t}, j=7.2 \mathrm{~Hz}$. 3H). $2.59-2.62$ (m. 2H). 2.69-2.72 (m. $2 \mathrm{H}$ ). 4.08 (q. $J=7.2$ Hz. 2H). 7.14 (s. lH). $7.2 \mathrm{l}$ (d. $J=8.6 \mathrm{~Hz} .2 \mathrm{H}$ ). 7.33 (d. $j=8.6 \mathrm{~Hz} .2 \mathrm{H}) . \mathrm{Z}$-form: ${ }^{1} \mathrm{H} \mathrm{NMR}\left(\mathrm{CDCl}_{3}\right) \delta 1.18(\mathrm{t} . J=7.2$ Hz. $3 \mathrm{H}) .2 .56-2.66(\mathrm{~m} .4 \mathrm{H}) .4 .08(\mathrm{q} . J=7.2 \mathrm{~Hz} .2 \mathrm{H}) .6 .91$ (s. $1 \mathrm{H}) .7 .30(\mathrm{~d} . J=8.4 \mathrm{~Hz}, 2 \mathrm{H}) \cdot 7.58(\mathrm{~d} . J=8.4 \mathrm{~Hz}$. $2 \mathrm{H}$ ): Mass (70 eV) $m z$ (rel. intensity) 127 (27). 140 (29). $1.54(100), 176(31), 189(75), 263$ (M'. 28). 265 $\left(\mathrm{M}^{-}+2,9\right)$.

Acknowledgments. This work was supported by a Korea Research Foundation grant (KRF-2002-015-CP(0215).

\section{References and Notes}

1. (a) Basavaiah. D.: P'andiaraju. S. Tetwhedon Lett. 1995. 36. 757. (b) Basavaiah. D.: Pandiaraju. S.: Krishnamacharyulu. M. Symlett 1996. 747

2. (a) Shen, Y.: 7.hang. 7. Symit. Commun. 2000, 30. 445. (b) Shen. Y.; 7.hang, 7. J. Chem. Res. Sf 1999. 556.

3. Im, Y. J.: Kim, J. M.: Kim. J. N. Bull. Aonum Chem. Soc. 2002. 23. 1361 .

4. (a) Taber. D. F.: Amedio. J. C. Jr.: Gulino. F.J. Org. (hem. 1989. 54. 3474. (b) Taber. D. F.: Amedio. J. C. Jr.: Patel. Y. K. J. Org. Chem 1985, 50, 3618. (c) Miles. D. H.: Huang. B.-S. J. Ory. Chem 1976, H, 208. (d) Huang. B.-S.: Parish. F. I.: Miles. D. H. J. Org. Chem. 1974. 39.2647.

5. Laragoza. F. J. Org. (Yhem, 2002. 67. 4963.

6. Johnson. A. W.: Kaska. W. C.: Strazew ski. K. A. O.: Diron. D. A. Flides and Inines of Phosphorws. John Wiley \& Sons. Inc.: 1993: pp $166-178$.

7. (a) Kim, I. N.: I.ce, K. Y. Cm Omg Chem 2002, 6, 627. (b) Im, Y. J.: I.ce, K. Y.: Kim, T. II.: Kim, I. N. Fetrohedron Lett. 2002. 43. 4675. (c) Kim. J. N.: Chung. Y. M.: Im. Y. J. Tetratedron Lett. 2002. 13. 6209. (d) Kim. J. N.: Im. Y. I.: Kim. J. M. Tetrathedmot lett. 2002. 43. 6597. (e) Kim. J. N.: Lee. H. J.: (jong. J. H. Tetratredron Leth. 2002. 43.9141. (t) Kim, J. N.; I.ce. H. I.; I.ce, K. Y.: Gong. I. II. Swltet/ 2002. 173. (g) Gong. J. H.: Im, Y. J.; I.ce, K. Y.: Kim, I. N. Tetrohedron Letl. 2002. f3. 1247. (h) Chung. Y. M.: Gong. J. H: Kim. T. H.: Kim. J. N. Tt'tratedm lett. 2001. t2. 9023. (i) Kim. J. N.: Kim. H. S.: Gong. J. H.: Chung. Y. M. Tetrahedwon Iett. 2001. +2.8341. (j) Kim. J. N.: Im. Y. I.: Gong. J. H.: I ce. K. Y. Terrohedron Le'll 2001. 42, +195. (k) Kim, I. N.: I.ee, II. I.: I ce, K. Y.: Kim. II. S. Tetrohedrom Leff. 2001, 42. 3737. (1) Kim, II. S.: Kim, T. Y.: I.ee, K. Y.: Clung. Y. M.: Lee. H. I.: Kim. J. N. Tetrahedryt Teft. 2000. HI. 2613. (m) Kim. J. N.: Lee. K. Y.: Kim. H. S.: Kim. T. Y. Org. le'tt. 2000.? 343. (11) Lee. H. J.: Seone. M. R.: Kim. J. N. Tetrahtedron Iett. 1998, 39. 6223. (o) J.ec. H. J.: Kim, II. S.: Kim, J. N. Tetrahedron Letf. 1999, t0, 4.36.3. (p) Chung. Y. M.: I.e., I. I.: I Inang. S. S.: Kim, I. N. Bull Konem Chem Soc 2001, 22, 799. (q) Im. Y. J.;

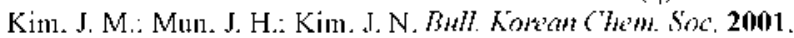
22. 349 